\title{
Filigrane
}

Écoutes psychanalytiques

\section{Névroses actuelles, solution somatique ou mélancolique}

\section{François Duparc}

Volume 24, numéro 1, printemps 2015

Le devenir de la psychanalyse. Échos d'ici et d’ailleurs...

URI : https://id.erudit.org/iderudit/1033082ar

DOI : https://doi.org/10.7202/1033082ar

Aller au sommaire du numéro

Éditeur(s)

Revue Santé mentale au Québec

ISSN

1192-1412 (imprimé)

1911-4656 (numérique)

Découvrir la revue

Citer cet article

Duparc, F. (2015). Névroses actuelles, solution somatique ou mélancolique. Filigrane, 24(1), 43-52. https://doi.org/10.7202/1033082ar

\section{Résumé de l'article}

Face aux pathologies actuelles et au spectre de la dépression dans ses multiples formes, l'idée d'une solution mélancolique ou somatique invite à répondre à un défi nosologique, un peu négligé dans le contexte de la psychiatrie contemporaine. Il s'agit de remettre à l'ordre du jour les névroses actuelles de Freud, pour complexifier nos stratégies d'analystes. De différencier l'ombre d'un objet idéalisé retombant sur le moi, d'une carence représentative traumatique telle qu'elle va produire une « forclusion » du fantasme originaire de castration, à l'origine d'une lignée psychosomatique proche de la neurasthénie ou de la dépression essentielle. Un exemple clinique, avec une stratégie adaptée pour une personne ayant développé une pathologie cancéreuse, permettra d'illustrer ces notions. La stratégie employée, qui vise à travailler avec des traces mal représentées, n'est pas incompatible avec l'évolution vers une analyse classique, en déjouant le piège de la névrose actuelle de notre temps. 


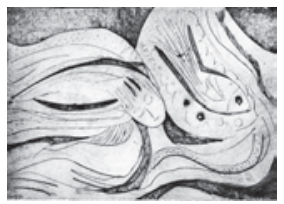

Névroses actuelles,
solution somatique
ou mélancolique

François Duparc

Face aux pathologies actuelles et au spectre de la dépression dans ses multiples formes, l'idée d'une solution mélancolique ou somatique invite à répondre à un défi nosologique, un peu négligé dans le contexte de la psychiatrie contemporaine. Il s'agit de remettre à l'ordre du jour les névroses actuelles de Freud, pour complexifier nos stratégies d'analystes. De différencier l'ombre d'un objet idéalisé retombant sur le moi, d'une carence représentative traumatique telle qu'elle va produire une «forclusion" du fantasme originaire de castration, à l'origine d'une lignée psychosomatique proche de la neurasthénie ou de la dépression essentielle. Un exemple clinique, avec une stratégie adaptée pour une personne ayant développé une pathologie cancéreuse, permettra d'illustrer ces notions. La stratégie employée, qui vise à travailler avec des traces mal représentées, n'est pas incompatible avec l'évolution vers une analyse classique, en déjouant le piège de la névrose actuelle de notre temps.

e psychanalyste, de nos jours, est de plus en plus confronté aux pathologies de «l'actuel» au double sens de l'agir et de l'instantané. Parmi celles-ci les réactions dépressives à des atteintes «économiques» (au sens de l'économie psychique) et à des traumatismes sont évidemment des plus fréquentes. Mais si on veut ne pas se restreindre au seul symptôme, qui est souvent passager, masqué ou trompeur, il est important de disposer d'une approche un peu nosologique qui ne se limite pas aux apparences, au «contenu manifeste», comme Freud le disait pour les rêves.

Ainsi, l'idée d'une «solution» mélancolique ou somatique au spectre de la dépression est une invitation à envisager les différentes réponses possibles à un facteur traumatique. Ce dernier donne un caractère d'urgence ou d'actualité à la pathologie qui en résulte, surtout s'il est amplifié par la résonance de multiples après-coups. Il va aboutir soit à un investissement pulsionnel plus ou moins fixé, soit à sa décharge selon les lois de la seconde topique de Freud. Puis, dans certains cas, ce facteur traumatique va se manifester par une pathologie narcissique limite, ou à défaut par une pathologie psychosomatique. Mais le psychanalyste, gardien du temps à long terme, ne 
doit pas négliger l'histoire à reconstruire, au-delà de la souffrance actuelle, afin de déjouer la compulsion de répétition qui se cache derrière l'actualité des symptômes.

À l'ère du DSM V, les psychanalystes ont en effet un défi à relever, celui d'une nosologie psychanalytique différente de la dichotomie névrose/psychose, des stades pulsionnels, ou du fourre-tout des états-limites. Le DSM, névrose actuelle de la psychiatrie, écrase la temporalité à long terme et les structures psychopathologiques derrière le «trouble» ou le symptôme. Son but est de permettre aux médecins un diagnostic rapide, des thérapies brèves, et la prescription systématique de médicaments psychotropes. Mais cette classification prolonge ainsi le malaise dans la culture qui pousse à une «modernité sans histoire» (M. Corcos); ainsi, pour la dépression, la différence avec un deuil normal s'est amenuisée d'une édition du DSM à l'autre: dans la dernière, une dépression cesse d'être mineure après trois semaines!

Les psychanalystes ne peuvent pas pour autant, dans une réaction de rejet, renoncer à la nosologie dont ils ont besoin pour soutenir le cadre à proposer (ils disposent aujourd'hui de nombreux aménagements, de la cure classique à la relaxation psychanalytique ou au psychodrame), et pour les guider dans leur stratégie interprétative — associative, narrative ou constructive. Une vraie nosologie, à l'opposé du DSM, comporte une phase de diagnostic étiologique, qui doit précéder le traitement.

Deux idées de Freud méritent d'être reprises pour améliorer notre nosologie. Tout d'abord la notion de névrose actuelle, qu'il a introduite en 1896. Le terme de névrose actuelle est bienvenu, en français, car il évoque un double sens: une «névrose de l'actuel», et une "névrose de l'acte (ou de l'agir)». Dans les deux cas, l'absence de capacité à différer la décharge abrase toute temporalité, du fait d'une carence de représentations, et la dominance de l'excitation sur la pulsion pousse à l'agir en court-circuitant l'activité de jugement. Freud avait décrit en 1898 (La sexualité dans l'étiologie des névroses) deux névroses actuelles, pour les opposer aux psychonévroses: la neurasthénie, et la névrose d'angoisse. Il ajoutera l'hypocondrie en 1914. Il aurait même pu ajouter la toxicomanie, s'il n'en avait pas souffert avec la cocaïne et le cigare... Certaines formes de psychoses (expérience délirante primaire, dépersonnalisation, psychose blanche) pourraient aussi constituer une cinquième lignée.

La notion de névrose actuelle rejoint ce que Freud développe en 1926 (dans Inhibition, symptôme et angoisse) avec la névrose traumatique. Elle est la tache aveugle, l'irreprésentable ou le grain de sable, actualisation après-coup 
d'une inscription traumatique qui en est restée, du fait du débordement économique, au stade primaire de la forme sensori-motrice - le premier des trois stades de la représentation, rappellent R. et $\mathrm{S}$. Asseo, en reprenant l'Esquisse de Freud.

L'habillage psychique de l'infantile, dit D. Scarfone, ne fait que recomposer l'indicible du sujet infans, et ne peut être saisi que par l'imitation, les images motrices du partenaire. Les images sensori-motrices et leurs résonances émotionnelles vont être alors contenues par les images produites par le travail du rêve. Mais si le cauchemar l'emporte, «si la quantité d'excitation ou l'impréparation empêchent la liaison, la situation se détériore, endommageant l'appareil psychique lui-même à qui il ne reste alors que la voie de la décharge, soit vers l'extérieur, et c'est l'agir, soit vers l'intérieur du corps, et c'est la somatisation» (D. Scarfone, 2014, p. 374). J'ajouterai que la résonance des traumas aux différentes crises des sept âges de la vie - naissance, marche, Edipe, puberté, maternité, andropause, ménopause - à ces âges où la quantité d'excitation pulsionnelle déborde la capacité de liaison, est souvent à l'origine du déclenchement des névroses actuelles (traumatiques), comme des grains de sable autour desquels se construiraient les édifices pathologiques.

Pour enrichir notre nosologie, après les névroses actuelles, nous reprendrons la conception de lignées structurales, c'est-à-dire la palette des névroses possibles (hystérie, obsession, phobies), des états narcissiques-limites que Freud appelait névroses narcissiques (cyclothymie, paranoïa, perversions), et des lignées psychosomatiques décrites par M. Fain dans L'enfant et son corps (1970).

La notion de névrose actuelle fait référence à la qualité des représentations dont le sujet dispose pour contenir l'excitation traumatique qui peut se situer, dans une vision génétique des représentations, sur un continuum allant du grain de sable de la décharge comportementale, émotionnelle primaire et sensorimotrice, à la représentation imagée de chose, puis de mots. Mais la notion de lignées structurales nous fait plutôt envisager la diversité des figures possibles, depuis les formes primaires de l'émotion, du comportement et de la gestuelle, jusqu'à la diversité des fantasmes originaires qui composent l'Eedipe, en passant par les mécanismes du rêve, de l'image ou du symbole. Il s'agit d'un axe horizontal de classification nosologique, différent de l'axe vertical de la gravité des pathologies et des traumas qui vont de la névrose aux états-limites, jusqu'aux somatisations (la psychose pouvant être conçue comme une pathologie psychosomatique). 
Freud n'avait pas évoqué l'idée de lignées. Par contre, il avait décrit les différents fantasmes originaires composant l'CEdipe: trois, au début: séduction, castration, scène primitive. Puis plus tard (dans une note de L'homme aux loups) le fantasme de retour au ventre maternel. Dès le petit Hans, il avait perçu le sujet comme pris dans le désir parental et la structure familiale œedipienne. Les fantasmes prennent corps durant les premiers mois, par les rites de maternité, de naissance et d'accueil, dans les modes de portance et de holding, la «chanson de gestes» ou la «danse interactive» (D. Stern) entre la mère et son bébé. Très tôt également, dès fin de la première année, la «censure de l'amante» (M. Fain) du couple parental va se mettre en place. Le sujet se voit ainsi doté dès l'origine d'un matériel de base, d'un «kit de construction» pour son futur complexe. Mais ce matériel doit être élaboré et perdre sa violence, pour ne plus faire le jeu de la pulsion de mort: le sujet pourra ainsi dépasser ses «fantasmes originaires de mort», pour les transformer en des fantasmes originaires de vie, sous l'instance protectrice du Surmoi-Idéal, à condition que cette instance soit l'héritage bien élaboré du couple parental.

Une remise en question actuelle du complexe d'Edipe prétend que ce roman familial concerne surtout les névrosés, qui organisent leurs fantasmes autour de la sexualité parentale et des interdits à leur sujet, mais que nos patients actuels sont moins névrosés que ceux de Freud. Ainsi, dans un numéro récent de la revue Filigrane, l'idée proposée était que les analystes recevaient aujourd'hui peu de patients porteurs de structures œedipiennes classiques, mais plutôt des pathologies narcissiques limites. J'ai répondu qu' "Edipe court toujours», de nos jours comme aux temps de la Grèce, d'autant que la légende d'CEdipe décrit l'histoire d'une pathologie comportementale, traumatique, peu œdipienne au sens névrotique du terme: un Cedipe pris dans l'agir et la névrose actuelle. La violence de l'exposition à la mort de l'enfant Eedipe, sa migration à la suite des révélations de Tirésias sur ses origines, la rencontre avec la Sphinge, le meurtre de son père sur la route et son histoire incestueuse, jusqu'à son auto mutilation castratrice finale, tout cela relevait d'une sérieuse carence narcissique et d'une violence peu élaborée, sinon en après-coup, par Sophocle. Ces agirs, dans la version originaire du mythe grec, témoignent d'un manque d'élaboration des représentations psychiques, comme aux débuts de la vie, sous l'effet de la violence d'un traumatisme, lors d'un épisode catastrophique qui déborde les capacités du pare-excitations, ou plus tard, du fait d'une résonance entre des traumatismes cumulatifs, à l'origine d'états-limites ou de pathologies psychosomatiques. 
Ainsi, l'inscription traumatique d'un des fantasmes originaires sous une forme primaire (émotionnelle ou sensori-motrice) — sa «forclusion» dans le registre des représentations de choses ou de mots - est à l'origine d'une lignée psychosomatique. Mais la gravité de l'affection dépend de l'importance du noyau traumatique irreprésentable sous forme d'images ou de fantasmes élaborés dans le registre de l'Edipe.

Freud a décrit, je le rappelais ci-haut, quatre fantasmes originaires: séduction, castration, scène primitive et plus tard retour au ventre maternel. Auxquels j'ajouterais, fidèle à son esprit, un cinquième, le fantasme de meurtre cannibalique, si présent dans l'CEdipe que Freud ne l'a même pas compté, car il était pour lui au centre de l'héritage phylogénétique du complexe. Au total, on peut donc décrire cinq lignées psychosomatiques. Mais si on s'intéresse aux solutions mélancolique ou de dépression somatique, le fantasme en cause est évidemment celui de castration. Ce fantasme, interdit protecteur dans sa version positive, à l'âge de l'Edipe proprement dit vers quatre ou cinq ans, vise à faire accepter les limites nécessaires au désir de l'enfant, le manque de la sexualité et l'attente nécessaire, soutenues par l'instance protectrice du couple Surmoi-Idéal.

Dans la dépression narcissique, ou mélancolie, l’ombre de l'objet perdu retombe sur le moi, dit Freud, ce qui relève plus de l'angoisse d'effondrement que de l'angoisse de castration. L'ombre est davantage celle d'un objet idéalisé, mal représenté, tel l'oculus dont parle D. Scarfone; ce n'est pas le reflet d'un objet oedipien, comme dans la dépression névrotique réactionnelle au deuil d'un objet bien représenté. Voici déjà longtemps (1989), j'avais parlé des «objets infinis» des mélancoliques, en notant que ces objets idéalisés étaient les substituts d'objets parentaux réduits à un Surmoi ou à un Idéal du Moi tyrannique, sans tierceïté. L'objet peu fiable, inconsistant, idéalisé à la façon d'un soleil noir, retombe alors sur le moi, après une explosion maniaque plus ou moins nette, mais dont l'aspect quasi orgastique a pour effet de vider la libido du moi. J'avais évoqué à l'époque la présence fréquente d'une pensée concrète, conventionnelle, quasi opératoire, jouant le rôle d'une carapace neutralisant les affects, avec le risque de déclenchement d'un cycle maniacodépressif en cas de franchissement de cet obstacle par le transfert ou par l'analyste. L'épisode maniaque jouait le rôle d'une projection dans le vide, d'une vidange de la libido précédant l'effondrement mélancolique. La castration, ici, sera celle du moi, dont la jouissance démesurée provoque l'effondrement.

Mais si on suit cette idée, quelle différence avec la dépression essentielle décrite par P. Marty en 1968, génératrice de pensée opératoire, et de 
somatisations diverses, notamment par dépression du système immunitaire? La dépression essentielle est une découverte précieuse de la psychosomatique française, qui mérite notre attention. S’agit-il d'une névrose actuelle, prolongement de la «neurasthénie» que Freud avait en tête au moment de l'Esquisse? La dépression essentielle serait-elle une névrose actuelle avec une composante traumatique importante, mais invisible? Elle aurait ainsi des manifestations bien plus discrètes que celles de la dépression narcissique mélancolique, car l'ombre de l'objet ne saurait dans son cas retomber sur le moi, faute d'un objet secourable aux moments des traumatismes cumulatifs dont le sujet a souffert, sans pouvoir faire autre chose que les décharger dans une «manie blanche» (F. Duparc 2006) ou une hyperactivité opératoire non contenue par une représentation d'objet, même idéalisée et confuse, comme dans la mélancolie.

Qui dit traumatisme invisible dit déni ou «forclusion» (J. Lacan) d'un signifiant fondamental (ici le deuil, la perte, la castration). Ce qui se traduit au niveau primitif des émotions primaires et de la sensori-motricité, du rythme musical de la gestuelle et de l'image du corps. Quelque chose de la «chanson de gestes» mère-bébé, de l'histoire parentale précoce (transgénérationnelle) n'a pas connu d'élaboration symbolique vers l'image, endeçà même du langage. La censure de l'amante, forme primaire de l'Edipe (M. Fain) n'a pu se faire sans exclure un signifiant fondamental, la préforme d'un fantasme originaire; le fantasme de castration sous sa forme primaire sensori-motrice de chute ou d'abandon. L'effet cumulatif de deuils occultés au cours de la vie va accroître la forclusion. Mais la tristesse, le deuil, la dépression, sont invisibles dans la dépression essentielle et ne se manifestent pas sous une forme émotionnelle perceptible. Tout au plus une fatigue, un épuisement. L'effacement des affects ne permet pas l'expression d'une tristesse, d'une culpabilité ou d'une douleur psychique.

\section{Un bref exemple clinique}

Une femme dans la cinquantaine me consulte pour son mari qui vient d'avoir un léger accident vasculaire cérébral. Son idée est qu'il viendra peutêtre avec elle pour discuter de ses problèmes avec son employeur, qui refuse de prendre en compte son surmenage et continue de l'expédier sans cesse à l'étranger. Il refusera finalement de venir, mais elle continuera, parce qu'on lui annonce un cancer du pancréas qui ne devrait lui laisser que peu de temps à vivre. Elle croit cependant le médecin qui lui dit qu'avec la chimiothérapie et les interventions sur ses métastases, elle a une chance de guérir. 
J'accepte de l'accompagner durant les quatre ans qui vont lui rester à vivre — un temps plus long que ce que j'imaginais — jusqu'à une récidive qui l'emportera en quelques semaines.

Elle me décrit son travail, sa vie. Elle est la troisième de trois filles, et elle a appris récemment qu'elle n'était pas désirée par sa mère, très critique à son égard, sans aucune tendresse. Celle-ci, atteinte d'un début de démence, lui a confié cela alors qu'elle ne lui avait jamais fait de confidences. Parmi les causes possibles de sa dépression masquée, j'apprendrai aussi le départ de sa fille unique pour l'étranger comme enseignante. Avec les absences fréquentes de son mari, cela fait beaucoup. Elle se sent abandonnée, mais ne le montre jamais, assure son travail et son rôle de soutien de ses parents âgés, sans la moindre plainte.

J'apprends qu'elle a fait une analyse de plusieurs années avec un analyste classique dans ses interprétations sur son CEdipe, son lien exagéré avec son père, et sa rivalité avec sa mère. Elle y était venue par une amie de travail, pour des problèmes sexuels avec son mari, dont elle se culpabilisait. Durant toutes ces années, elle n'a jamais pleuré en séance. Elle rêvait peu, ce dont son analyste se plaignait, mais parlait de son métier, de son mari, de ses enfants, de façon très appliquée. Malgré tout, je n'avais pas l'impression d'une pensée opératoire, étant donné son mode d'association. On sait que pour C. Smadja, pensée opératoire et dépression essentielle sont liées, mais pour d'autres psychosomaticiens tels que Marty ou de M'Uzan, il s'agit probablement d'une autre lignée, celle des auto calmants du comportement ou de la pensée. Par contre, sa capacité à ressentir sa dépression était clairement défaillante: elle savait qu'elle avait toujours été la fille courageuse, celle sur qui on s'appuyait, et ne pouvait rien demander en retour.

Face à ce tableau, je lui propose une psychothérapie analytique corporelle (en face-à-face sur le divan) afin de l'aider à exprimer ses émotions, et à ressentir davantage son corps. Elle avait en effet, comme dans la neurasthénie, des sensations de vertiges et des accès de fatigue qui avaient précédé sa maladie. Dans les séances, je serai attentif aux ébauches tonico-émotionnelles dans son comportement, ce qui va l'aider à en prendre conscience et à se libérer. Elle me parle de sa mère et du manque de tendresse de sa part dont elle n'avait jamais parlé dans sa première analyse. Je fais néanmoins attention à ne pas la laisser aller trop vite. Lorsque je lui demande de sentir son corps sur le divan, elle réalise qu'elle a souvent l'impression de tomber. Au bout de quelques mois elle peut me raconter un premier cauchemar; elle rêve qu'elle a perdu sa fille de dix ans et qu'elle la voit au balcon 
d'un immeuble, prête à tomber pour la rejoindre. Elle se réveille avec une impression de chute. Ce rêve va lui permettre de pleurer pour la première fois.

Nous aurons d'autres moments forts dans ce travail analytique auquel elle va fortement s'accrocher, car, me dit-elle, il lui permet de donner un sens à des choses qu'elle faisait uniquement par devoir. Elle va découvrir combien elle avait enfermé en elle toute sa colère contre sa famille où l'on ne parlait jamais des émotions, ni de la sexualité. Une récidive se produira à un moment douloureux pour elle; sa mère doit être placée dans un centre pour sa démence, et elle s'en sent très coupable. À cette occasion, le neuropsychiatre qu'elle consulte avec sa mère, qui a le DSM pour bible, va lui proposer de lui donner à elle aussi des médicaments, des antidépresseurs en l'occurrence, car il trouve que son état dépressif réactionnel dure trop longtemps (plus d'un mois). Mais elle décline sa proposition, assez en colère, ce qui est nouveau pour elle. Pour moi au contraire, c'était une chance qu'elle ait pu ainsi pleurer et se préparer au deuil de sa mère, même si cela ne lui garantissait pas l'absence de récidive pour elle-même. Cela lui permettra aussi de se rapprocher de son mari et de ses enfants, qui vont venir la soutenir, ce qu'ils ne faisaient pas jusque-là, et ce qui lui sera précieux pour la suite de l'évolution de sa maladie.

Le travail avec cette patiente, malgré ses difficultés, aura eu aussi le mérite de m'encourager à consolider mon approche de la relaxation psychanalytique (inspirée d'Ajurriaguerra, distincte de la relaxation opératoire et magique habituelle) comme un travail en après-coup de cures plus traditionnelles mais insuffisantes, et de favoriser le passage à une cure classique, après reconstruction d'une histoire traumatique mal élaborée.

\section{En guise de conclusion}

Pour en revenir à la question des névroses actuelles et de la nosologie psychanalytique, je pense que le but d'une nosologie est de permettre à l'analyste une stratégie qui ne soit pas seulement à court terme, mais qui puisse évoluer au cours du temps, avec une adaptation du cadre psychanalytique qui ne néglige pas les autres potentialités structurales du sujet, et les après-coups constructeurs de son histoire (comme A. Green l'a évoqué dans Le temps éclaté). Car une fois passé le temps de la dépression, les noyaux hystériques, phobiques ou obsessionnels, les pathologies addictives ou perverses, les autres fantasmes originaires de l'CEdipe vont se remettre en place, et nécessiter de nouvelles stratégies interprétatives, voire reconstructives. 
La crise de la psychanalyse contemporaine face aux thérapies comportementales ou cognitives, se caractérise par la contraction de la temporalité qui altère notre vision de l'humain, répondant par l'urgence et par une consommation immédiate aux demandes et aux troubles - ce que j'ai appelé l'idéologie de la communication et de la consommation (F. Duparc 2004). Elle a aussi pour effet de pousser parfois les analystes à une attitude extrême inverse, un rejet de toute nosologie, qui n'est pas moins néfaste que la pauvreté nosologique des classifications psychiatriques.

D'autant que le rejet de la psychanalyse, après un âge d'or dominé par la psychologie du Moi et des névroses, est sans doute lié à un développement insuffisant, à ses débuts, de la compréhension des pathologies narcissiques et limites, ainsi qu'au fait qu'elle ne tenait pas compte de l'adaptation nécessaire de la technique à ces pathologies (comme dans le cas de ma patiente, sa première analyse trop «classique»). Notamment la difficulté des analystes à accueillir le corps, les émotions et les agirs comportementaux comme matériel de libre association, au même titre que la parole. Une nosologie adaptée, puis une stratégie s'en inspirant, permettent un cadre «sur mesure» pour ces pathologies-limites qui ne disposent pas de représentations suffisantes, mais un matériel «blanc» de l'ordre de l'hallucination négative.

Ces techniques, autrefois dénommées péjorativement "psychothérapies d'inspiration psychanalytique» que sont le psychodrame, l'analyse de l'enfant par le jeu, ou la relaxation psychanalytique, dans lesquelles les états du corps, les tensions, les postures et les émotions font partie de l'association libre, sont aux confins de l'agir et du somatique, mais restent authentiquement psychanalytiques. Elles n'obligent pas, bien au contraire, à renoncer à la construction de l'histoire dans ses différentes versions, et à une évolution vers une cure analytique classique. Ainsi les pièges de l'actualité, de la névrose actuelle ou traumatique (la dépression blanche, pour la lignée de l'inscription traumatique de la castration, et la solution mélancolique ou somatique), ne se refermeront pas sur le sujet ni sur son analyste dans une compulsion de répétition mortifère. Ce dernier ne se limitera pas à la cure immédiate des symptômes, mais aura aussi à cour de laisser leur place aux autres buts de la psychanalyse; faire un deuil, découvrir la séduction de la nouveauté, aimer et travailler de façon créative, trier les acquis de son héritage et de son histoire pour en faire son propre devenir.

François Duparc dr.francois.duparc@wanadoo.fr 


\section{Références}

Asséo, R. et S. Dreyfus-Asséo, S. (2014). L'actuel en psychanalyse, Rapport du Congrès des psychanalystes de langue française à Montréal. Revue française de psychanalyse, 67 (5).

Corcos, M. (2011). L'homme selon le DSM, le nouvel ordre psychiatrique. Paris: Abin-Michel.

Dechaud-Ferbus, M. (2011). Cet autre divan, psychanalyse de la mémoire du corps. Paris:

Presses universitaires de France.

Cupa, D. et Pirlot, G. (2012). André Green, Les grands concepts de la psychanalyse. Paris: Presses universitaires de France.

Duparc, F. (1989). Les objets infinis du mélancolique. Revue française de psychanalyse, 53 (1), 481-486.

Duparc, F. (1992). Nouveaux développements sur l'hallucination négative et la représentation. Revue française de psychanalyse, 56 (1), 101-121.

Duparc, F. (2004). Le mal des idéologies. Paris: Presses universitaires de France.

Duparc, F. (2006). Deuils invisibles, manie blanche et clivages; facteurs de risque cancéreux? Revue française de psychosomatique, 30 (2), 79-100.

Duparc, F. (2008). Ils se marièrent et eurent beaucoup d'enfants; sur les fins d'analyse. Revue française de psychanalyse, 72 (1), 115-127.

Duparc, F. (2010). Edipe court toujours, Filigrane, 19 (2), 59-72.

Duparc, F. et Pichon, M. (dir.) (2010). Jacques Lacan, au fil du miroir. Paris: In Press.

Fain, M. (1970). L'enfant et son corps. Paris: Presses universitaires de France.

Fain, M. et Duparc, F. (2006). La censure de l'amante. Paris: Delachaux et Niestlé.

Green, A. (2000). Le temps éclaté. Paris: Presses universitaires de France.

Marty, P. (1968/1995). La dépression essentielle. Revue française de psychosomatique, 8, 209213.

Scarfone, D. (2014). L'impassé. L'actuel en psychanalyse. Rapport du Congrès des psychanalystes de langue française à Montréal. Revue française de psychanalyse, 78 (5), 1357-1428.

Smadja, C. (2008). Les modèles de la psychosomatique. Paris: Presses universitaires de France 